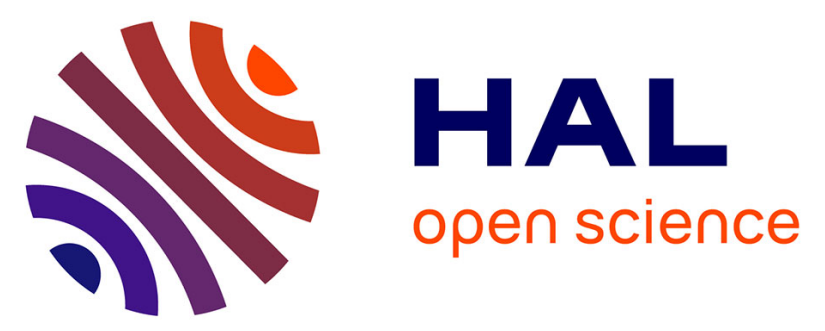

\title{
The effects of electronic impurities and electron-hole recombination dynamics on large-grain organic-inorganic perovskite photovoltaic efficiencies
}

Jean-Christophe Blancon, Wanyie Nie, Amanda J. Neukirch, Gautam Gupta, Sergei Tretiak, Laurent Cognet, Aditya D. Mohite, Jared J. Crochet

\section{To cite this version:}

Jean-Christophe Blancon, Wanyie Nie, Amanda J. Neukirch, Gautam Gupta, Sergei Tretiak, et al.. The effects of electronic impurities and electron-hole recombination dynamics on large-grain organicinorganic perovskite photovoltaic efficiencies . Advanced Functional Materials, 2016, 26 (24), pp.4283. 10.1002/adfm.201505324 . hal-01390068

\section{HAL Id: hal-01390068 https://hal.science/hal-01390068}

Submitted on 31 Oct 2016

HAL is a multi-disciplinary open access archive for the deposit and dissemination of scientific research documents, whether they are published or not. The documents may come from teaching and research institutions in France or abroad, or from public or private research centers.
L'archive ouverte pluridisciplinaire HAL, est destinée au dépôt et à la diffusion de documents scientifiques de niveau recherche, publiés ou non, émanant des établissements d'enseignement et de recherche français ou étrangers, des laboratoires publics ou privés. 


\section{WILEY-VCH}

DOI: 10.1002/ ((please add manuscript number))

Article type: Full Paper

\section{The effects of electronic impurities and electron-hole recombination dynamics on large- grain organic-inorganic perovskite photovoltaic efficiencies}

Jean-Christophe Blancon, Wanyi Nie, Amanda J. Neukirch, Gautam Gupta, Sergei Tretiak, Laurent Cognet, Aditya D. Mohite and Jared J. Crochet*

Dr. J.-C. Blancon, Dr. J. J. Crochet

Los Alamos National Laboratory, Physical Chemistry and Applied Spectroscopy, Los Alamos, New Mexico 87545, USA.

E-mail: jcrochet@lanl.gov

Dr. W. Nie, Dr. G. Gupta, Dr. A. D. Mohite

Los Alamos National Laboratory, Materials Synthesis and Integrated Devices, Los Alamos, New Mexico 87545, USA.

Dr. A. J. Neukirch, Dr. S. Tretiak

Los Alamos National Laboratory, Theoretical Chemistry and Molecular Physics, Los Alamos, New Mexico 87545, USA.

Dr. L. Cognet

Univ. Bordeaux, Laboratoire Photonique Numerique et Nanosciences, UMR 5298, F-33400

Talence, France.

Institut d'Optique \& CNRS, LP2N UMR 5298, F-33400 Talence, France.

Keywords: carrier dynamics, electronic impurities, organic-inorganic perovskite, photovoltaic.

Organometallic perovskites have attracted considerable attention after promising developments in energy harvesting and other optoelectronic applications. However, further optimization will require a deeper understanding of the intrinsic photo-physics of materials with relevant structural characteristics. Here we investigate the dynamics of photogenerated charge carriers in large-area grain organometallic perovskite thin films via confocal time-resolved photoluminescence spectroscopy. It is found that the bimolecular recombination of free charges is the dominant decay mechanism at excitation densities relevant for photovoltaic applications. Bimolecular coefficients are found to be on the order of $10^{-9} \mathrm{~cm}^{3} / \mathrm{s}$, comparable to typical direct-gap semiconductors, yet significantly smaller than theoretically expected. We also demonstrate that there is no degradation in carrier transport in these thin films due to electronic impurities. 


\section{WILEY-VCH}

Suppressed electron-hole recombination and transport that is not limited by deep level defects provide a microscopic model for the superior performance of large-area grain hybrid perovskites for photovoltaic applications.

\section{Introduction}

Direct band gap semiconductors with low defect densities, such as single crystalline GaAs, are the epitome of high efficiency optoelectronic devices. However these materials are obtained using expensive high temperature crystal growth techniques such as molecular beam epitaxy. Consequently, there has been a constant search over the last two decades for new materials that can be obtained using scalable solution based strategies. However most solutionprocessed materials are plagued with polydispersity, lack of crystallinity, and unacceptable levels of electronic defects. Over the past few years, solution-process organometallic perovskite semiconducting materials (mainly $\mathrm{CH}_{3} \mathrm{NH}_{3} \mathrm{PbI}_{3}$ and $\mathrm{CH}_{3} \mathrm{NH}_{3} \mathrm{PbI}_{3-\mathrm{x}} \mathrm{Cl}_{\mathrm{x}}$ ), which promise low-cost solution processing together with favourable intrinsic properties for optoelectronic applications, have attracted a significant research effort after initial demonstrations of promising performances in photovoltaics ${ }^{[1,2]}$, light emitting diodes ${ }^{[1,3]}$, lasing ${ }^{[4-6]}$. However, several challenges remain towards further improvements of performance, reproducibility, and reliability of these devices

under continuous operating conditions ${ }^{[1]}$. Addressing those matters, inevitably, requires a fundamental understanding of the intrinsic photo-physical properties of bulk perovskites at conditions relevant for application.

Up to date, the details of charges carrier dynamics in organometallic perovskites are still under debate. More precisely, there is a lack of understanding of local intrinsic kinetics of photoexcited carriers in perovskite thin films, serving as active layers in solar cells, at device operating conditions. This knowledge requires replicating solar illumination excitation densities under 


\section{WILEY-VCH}

broadband spectral excitation. Indeed, most investigations of light emission have been conducted at narrow spectral bandwidth, which may not capture the larger picture relevant for practical solar cell devices. Furthermore, due to sample inhomogeneities, local spatial probing of optical properties is the best approach to relate structural characteristics to charge carrier dynamics. In particular, for future improvements of device performance, it is important to decipher the dynamics of charge carriers in organometallic perovskites at photo-excitation densities close to operating conditions at the microscale.

Despite several attempts to correlate the optical properties and photo-excited carrier dynamics within a given material morphology ${ }^{[2,7-9]}$, only a few reports have explored optical properties at the micro-scale or at the single grain size in thin films for practical applications ${ }^{[2,9]}$. Two of the major hindrances to such studies have been low crystalline quality and small grain sizes. On the other hand, there have been several attempts to investigate the optical properties and dynamics of carriers in bulk perovskite thin films, which consists of ensembles of grains. Typically this is performed at room temperature, using light emission ${ }^{[2,10-18]}$ and absorption $^{[11,14,19-23]}$ characteristics to develop photophysical models. The first set of studies, hypothesizing a mixed response of excitonic and free-carrier photo-excitations, suggested extraordinary charge carrier diffusion lengths derived from nearly mono-exponential photoluminescence decays ${ }^{[10,11]}$. Others have observed mixed monomolecular and bimolecular mechanisms which were attributed to the competition of trap assisted and free carrier recombination ${ }^{[12,14,15,18,20]}$. These results are in sharp contrast to what is expected in conventional semiconducting direct band-gap materials with high crystallinity, which at room temperature should display a pure bimolecular recombination of free charges carriers ${ }^{[24,25]}$ and have never 


\section{WILEY-VCH}

been observed. Monomolecular decay of photoluminescence is only observed when electronic defects or excitonic effects dominate.

The growth of organometallic perovskite crystals has recently reached a milestone with the synthesis by solution processing of thin films with large crystalline grain structures ${ }^{[2]}$. Moreover, these large grains have been reported to be free of deep level electronic impurities resulting in near intrinsic transport properties as well as stable and reliable solar cell performances. For these reasons, they offer a new opportunity to access the local intrinsic photophysical properties of organic-inorganic perovskite.

In this investigation, we unify the micro-scale dynamics of photo-carriers and macroscale solar cell performance of large grain methylammonium halide perovskite thin films. Most importantly, we demonstrate a pure bimolecular recombination through confocal time-resolved photoluminescence spectroscopy in large crystalline grains over a broad spectral range at excitation densities relevant for photovoltaic applications. This is a textbook signature of defectfree bulk direct band gap semiconducting materials, where the bimolecular recombination of photo-generated electrons and holes via radiative $\mathrm{e}^{[24,25]}$ and non-radiative ${ }^{[26]}$ decay are prevailing relaxation processes. By comparing bimolecular coefficients computed within the Langevin model and derived from photoluminescence decays, we infer that recombining photogenerated carriers do not lead to major losses in photovoltaic devices because of efficient charge extraction. Additionally, measurements of an operating solar cell device under open circuit conditions (where carriers are not extracted from the cell) demonstrate that bimolecular recombination remains the sole decay channel of photo-generated charges and any defects formed during device fabrication are minor. These results demonstrate that solution cast large-area organic-inorganic perovskites have a technological potential in optoelectronics comparable to conventional 


\section{WILEY-VCH}

semiconducting systems, and superb photovoltaic performance can be attributed to suppressed electron-hole recombination and negligible deep level electronic impurities.

\section{Results and Discussion}

\subsection{Unambiguous bimolecular recombination in large grain perovskites}

Thin films of crystalline grains of mixed halide perovskite $\mathrm{CH}_{3} \mathrm{NH}_{3} \mathrm{PbI}_{3-\mathrm{x}} \mathrm{Cl}_{\mathrm{x}}$ synthesized via a hot-casting method were used in this study ${ }^{[2]}$. The optical properties and carrier dynamics were investigated by micro-scale confocal spectroscopy and time-resolved photoluminescence (PL) which probes local regions in thin films used for solar cell applications (Note S1). The microscale approach locally interrogated, with micrometer resolution $\left(\sim 1 \mu \mathrm{m}^{2}\right)$, the optical response of a single large-area grains ( $>100 \mu \mathrm{m}$ in diameter) residing in a $\sim 300 \mathrm{~nm}$ thick perovskite thin film deposited on a glass substrate (Figure 1a, inset). The inner region of the large-area grain is relevant for photovoltaic applications as grain-boundaries in these large grains have a minor impact on the overall device performance ${ }^{[2]}$. Figure 1a shows the absolute absorption coefficient and normalized photoluminescence spectrum measured confocally within a single large-area grain. This was obtained by correcting for multiple reflections occurring at the air/film and film/substrate interfaces and we deduced an absorption coefficient $(\alpha)$ of $\sim 2.5 \times 10^{4} \mathrm{~cm}^{-1}$ at the band-edge in the near infrared that displays an increase of an order of magnitude over the visible spectral range. From these results we also extracted the real and imaginary parts of refractive index (Figure S1 and Note S2) demonstrating an acceptable agreement with our DFT calculations (Figure S2 and Note S3) and other recent studies ${ }^{[21-23,27]}$. The emission and absorption band-edge, observed at $1.626 \pm 0.002 \mathrm{eV}(763 \mathrm{~nm})$ and $1.62 \pm 0.01 \mathrm{eV}(765 \mathrm{~nm})$ respectively, display a relatively small Stokes shift at ambient conditions (Figure 1a). We emphasize the importance of determining the absolute absorption coefficient for correctly 


\section{WILEY-VCH}

interpreting the dynamics of carriers and the quantum efficiency of solar cell devices through accurate estimates of the carrier excitation density (Figure S1). Accurate determinations of $\alpha$ also allow for comparing the photo-excited carrier density $\left(N_{0}\right)$ achievable in our time-resolved photoluminescence (TRPL) experiment to $N_{0}$ obtained in a planar solar cell under standard AM1.5 global illumination. With our TRPL system, featuring a wavelength-tunable 6-ps-pulsedmonochromatic laser excitation at $1 \mathrm{MHz}$, one solar irradiance translates into a lower limit of $N_{0}=6.5 \times 10^{15} \mathrm{~cm}^{-3}$, and further reaching values above $8 \times 10^{15} \mathrm{~cm}^{-3}$ when considering the device structure geometry.

For quantitative understanding and measurements, time-resolved photoluminescence was then performed on the same grain as the absorption and photoluminescence measurements using time-correlated single-photon counting (see details in Note S1). Figure $1 \mathrm{~b}$ shows the timedependent photoluminescence intensity at the band-edge after excitation at $690 \mathrm{~nm}(1.80 \mathrm{eV})$ generating an equivalent excitation density of less than 4 suns of irradiance $\left(N_{0}=2.8 \times 10^{16} \mathrm{~cm}^{-}\right.$

${ }^{3}$ ). The decay kinetics was modelled with a numerical solution to the first order rate equation describing the recombination of valence and conduction band carriers coupled to a least squares optimization of the rate constant. Briefly, a focused laser pulse of photon energy $\hbar \omega$, described by the charge density generation rate $G=\frac{P}{S \hbar \omega} A \alpha$ (where $P$ is the laser power, $S$ is the spot size, $A$ the thin film absorbance), creates a non-equilibrium distribution carriers in the valence and conduction bands (see also Figure 4). These carriers quickly relax to the band-edge on a picosecond-time scale ${ }^{[11,20]}$, which is unresolved in our experiments. Subsequently, the recombination of carriers is modelled by a pure band-to-band or bimolecular recombination, $\dot{N}=$ $G-\gamma_{b} N^{2}$, of the non-equilibrium carrier density $N(t)$ (assuming equivalent photo-excited carrier density in the valence and conduction bands) and described by a single bimolecular 


\section{WILEY-VCH}

coefficient $\gamma_{b}$ that includes both radiative and non-radiative processes ${ }^{[24-26]}$. Here the intensity of light emission is given as: $I_{\mathrm{PL}} \propto N^{2}$, as will be demonstrated later. An example of the accuracy of this model is shown in Figure $1 \mathrm{~b}$ where an excellent fit is displayed for the only free parameter $\gamma_{b}=5.3 \times 10^{-9} \mathrm{~cm}^{3} / \mathrm{s}$. Interestingly, this bimolecular recombination coefficient is order(s) of magnitude larger than previous reports on smaller-grain-structured organometallic samples $^{[6,9,12,14,15,18,20]}$, and comparable to that of typical direct-gap semiconductors ${ }^{[28,29]}$. Again, this result attests both the crystalline quality of our samples and that the photo-excitation of free charge carriers is the dominating kinetic process. However, $\gamma_{b}$ is most likely influenced by photon recycling because of a small photoluminescence Stokes shift and a large absorption coefficient ${ }^{[25,30,31]}$. We therefore expect this value to be a lower bound of the recombination rate and further enhancements may be made in planar perovskite devices by limiting the angle of photon emission ${ }^{[32,33]}$. The determined recombination rate at this excitation energy $(\sim 1.8 \mathrm{eV})$ corresponds to an approximate $7 \mathrm{~ns}$ effective electron-hole recombination lifetime, and this result unambiguously demonstrates that free carriers, are the main photo-excitation in these materials. This is in agreement with recent reports of very small exciton binding energies (a few meV) at room temperature ${ }^{[21,34]}$, and possible ultra-fast exciton dissociations due to strong screening the electron-hole interaction by optical phonons and collective rotational motions of the organic cations $\left(\mathrm{CH}_{3} \mathrm{NH}_{3}\right)^{[35,36]}$.

\subsection{Density dependent emission intensity and dynamics}

Investigating the dependence of the recombination dynamics on the photoexcitation density is necessary to fully understand the relaxation processes of excess free charge carriers. In general, the photoluminescence intensity for electron-hole pair recombination is expressed as $I_{\mathrm{PL}} \sim N_{C} N_{V}$. Photoluminescence can also arise from recombination of an electron or hole with a charged 


\section{WILEY-VCH}

shallow defect such that the charge densities should be re-defined as $N_{C}(t)=N(t)+N_{u}$ in the case of n-doping and $N_{V}(t)=N(t)+N_{u}$ in the case of p-doping. The equilibrium free carrier density $N_{u}$, encompassing n-type or p-type unintentional doping, is related to intrinsic defects. Theoretical investigations have associated this type unintentional doping to mainly Frenkel-type point defects, including vacancies, interstitials, and substitutions, and demonstrated that defects with small formation energies are all shallow trap states due the ionic nature of the material ${ }^{[37,38]}$. In particular, donors and acceptors act as both a source of doping and recombination centres (non-radiative) for excess carriers. A donor (acceptor) is a shallow level impurity which has an energy level just below (above) the conduction (valence) bands and can easily transfer an electron (hole) to the band, such that $D^{0} \rightleftarrows D^{+}+e^{-}\left(A^{0} \rightleftarrows A^{+}+h^{+}\right)$. On the other hand, deep level impurities are trap states and are composed of defects with large formation energies, however are much lower in density. Moreover, Schottky-type, neutral vacancy pair defects of $\mathrm{PbI}_{2}$ and $\mathrm{CH}_{3} \mathrm{NH}_{3} \mathrm{I}$, are presumably abundant in solution-cast growth and are neutral de-localized states within the bands that do not contribute to doping. These defects are not expected to be major (non-radiative) recombination centres for carriers ${ }^{[37]}$. Our preliminary measurements of field-effect transistor responses, that will be reported elsewhere, suggests a slight n-type doping in thin films grown by the method above. Thus, in the case of defects in the conduction band, the time-dependent photoluminescence intensity becomes $I_{\mathrm{PL}}(\mathrm{t}) \sim N^{2}+N N_{u}$, and in the steady state limit $I_{\mathrm{PL}} \sim N_{0}^{2}+N_{0} N_{u}$. These two regimes of light emission in the steady state, free carrier and unpassivated defect assisted emission, were clearly identified by performing an excitation intensity dependent integrated photoluminescence study, Figure 2. This allowed for an estimate of an equilibrium density associated with unintentional doping due to intrinsic defects of $N_{u} \sim$ $2.5 \times 10^{15} \mathrm{~cm}^{-3}$. This is equivalent to $\sim 0.3$ sun irradiance. For an excitation density of $N_{0}>N_{u}$, 


\section{WILEY-VCH}

the regime in which solar cells operate, the photoluminescence intensity scales as $I_{\mathrm{PL}} \sim N^{2}$ where excess free carriers mostly undergo bimolecular recombination after having passivated the equilibrium background charge.

Following these steady state measurements, the dynamics of photo-excited carriers were investigated via TRPL over a relatively broad range of excitation densities at photon energy 1.8 eV (Figure 3). A general model of the carrier dynamics considering defect levels (trap states) probed by TRPL is given by the first-order rate equations:

$$
\begin{gathered}
\dot{N}=G-\gamma_{b}\left[x N^{2}+(1-x) N N_{u}\right]-\gamma_{\downarrow} N+\gamma_{\uparrow} N_{D} \\
\dot{N}_{D}=\gamma_{\downarrow} N-\gamma_{\uparrow} N_{D}-\gamma_{t} N_{D}
\end{gathered}
$$

where all quantities are defined in the schematics and captions of Figure 4, and $x$ (between 0 and 1) is determined from the static PL results (see previous paragraph and Figure 2). After photogeneration $(G)$, the excess of free carriers $(N)$ at the band-edge can: undergo bimolecular recombination $\left(\gamma_{b} N^{2}\right)$, exchange densities with trap states $\left(-\gamma_{\downarrow} N+\gamma_{\uparrow} N_{D}\right)$, and relax by undergoing trap-assisted non-radiative recombination $\left(-\gamma_{t} N_{D}\right)$; see also the schematic in Figure 2d. Here, we define the effective lifetime of free charge recombination $\tau_{b}=\left(\gamma_{b} N_{0}\right)^{-1}$, and characteristic lifetimes for the trap-assisted decay as $\tau_{\downarrow}=\gamma_{\downarrow}^{-1}$ and $\tau_{t}=\gamma_{t}^{-1}$. The exchange rates between the band-edge and trap states are directly related by the Boltzmann distribution defined from their energy splitting $\left(\Delta E_{D}\right): \gamma_{\uparrow}=\gamma_{\downarrow} \exp \left(-\frac{\Delta E_{D}}{k_{B} T}\right)$, where $k_{B}$ is the Boltzmann constant and $T$ the sample temperature $(295 \mathrm{~K})$. The TRPL kinetics were fit independently using a numerical solution to equations (1) (see results in Figure S3), yielding excellent fits with coefficients of determination $R^{2}$ exceeding 0.95 , with the exception of the kinetics associated with $N_{0}=$ $0.4 \times 10^{15} \mathrm{~cm}^{-3}$ for which $R^{2}=0.64$ due to low signal-to-noise ratio at low photon collection yields in these experiments. For an excitation density of $N_{0}<5 \times 10^{15} \mathrm{~cm}^{-3}$, the photoluminescence decays tend toward a more mono-exponential response mixed with the 


\section{WILEY-VCH}

bimolecular process of recombination in agreement with previous reports ${ }^{[10,12,14]}$; we label this as the "mixed regime". On the other hand, at higher excitation density, $N_{0}>5 \times 10^{15} \mathrm{~cm}^{-3}$, the kinetics yield a pure bimolecular recombination behaviour ("bimolecular regime"). Figure 3b,c present the photo-excitation density dependence of the trap-state energy level $\left(\Delta E_{D}\right)$, the maximum occupation of both the band-edge $\left(N^{\max }\right)$ and trap state $\left(N_{D}^{\max }\right)$, and the ratio $\tau_{\downarrow} / \tau_{b}$ deduced from the global fitting procedure. Moreover, following the high quality fits with equations (1), we hypothesize that Auger recombination is negligible in the range of excitation density explored here, $N_{0}<10^{18} \mathrm{~cm}^{-3}$, which is in agreement with the literature $\mathrm{e}^{[12,18]}$.

All parameters directly related to the nature of the trap state $N_{D}\left(\Delta E_{D}, N_{D}^{\max }, \tau_{\downarrow} / \tau_{\mathrm{b}}\right)$ present a clear change in behaviour at the threshold value $N_{\mathrm{th}} \sim 5.5 \pm 1.0 \times 10^{15} \mathrm{~cm}^{-3}$. This is identified as the photo-excitation density for which shallow trap states $\left(\Delta E_{D}<25 \mathrm{meV}\right)$ are filled thus becoming transparent to carriers. The effective defect density in our large-area grains is somewhat lower than previous report in bulk perovskite materials ${ }^{[14,15]}$ and comparable with a recent report on locally enhanced photo-emission in a micro-structured or Cl-enriched materials ${ }^{[9,39]}$. Also, $N_{\text {th }}$ is slightly larger than the unintentional doping density $N_{u}$ measured in the static regime, which suggests that either non-doping neutral defects act as non-radiative recombination centres or $\mathrm{n}$ - and p-type intrinsic defect states partially compensate the static PL signal while TRPL only probes defects related to the minority carriers ${ }^{[37,38]}$. Concomitant to the switching of the trap-assisted decay path from shallow trap states $\left(\Delta E_{D}<25 \mathrm{meV}\right)$ to deep trap states $\left(\Delta E_{D}>100 \mathrm{meV}\right)$, we observe a strong reduction of the maximum occupation of the defect level $N_{D}^{\max }$ as well as an increase of the time constant ratio $\tau_{\downarrow} / \tau_{b}$ above unity. The latter implies that the excess carriers preferentially decay through the bimolecular recombination pathway for $\tau_{\downarrow} / \tau_{b}>1$, where $\tau_{b}$ becomes the smallest effective lifetime. The former is 


\section{WILEY-VCH}

explained by the lack of carrier exchange between the band-edge and trap state per the Boltzmann distribution, where deep trap states should be unoccupied at room temperature (Figure S4). From this we describe a physical picture where for $\Delta E_{D}<25 \mathrm{meV}$, a carrier occupying $N_{D}$ decays non-radiatively at a rate $\gamma_{\mathrm{t}}$ but has also a chance to repopulate the bandedge with a rate $\gamma_{\uparrow}$, whereas, for $\Delta E_{D}>100 \mathrm{meV}$, a carrier has a negligible probability to transit from the trap-state to the bandedge $\left(\gamma_{\uparrow} \sim 0\right)$ and $\gamma_{\downarrow}$ is the rate dominating the trap-assisted decay pathway (see also Figure S3c,d).

To summarize these results, in the mixed regime $\left(\mathrm{N}_{0}<\mathrm{N}_{\mathrm{th}}\right)$ the photo-excited carriers decay by undergoing both trap-assisted non-radiative recombination (dominant mechanism) and bimolecular recombination, whereas in the bimolecular regime $\left(\mathrm{N}_{0}>\mathrm{N}_{\text {th }}\right)$ the former decay pathway is either strongly damped (weak decay to deep trap states) or totally prohibited (Figure 3d). The latter observation is in agreement with the low density of deep trap states in this

material, since they require much higher energy to be formed ${ }^{[37,38]}$. From the defect density we infer that large-area-grain structured solar cells operate in the bimolecular regime (at least in the spectral range around $1.8 \mathrm{eV}$ ) as reported in Figure 1b. This behaviour is different than previous studies reporting solar cell operation in the mixed regime involving trap-assisted recombination and bimolecular recombination to dark carriers due to doping ${ }^{[15,39]}$ and the pure bimolecular regime being reached at tens of solar irradiance $\mathrm{e}^{[15]}$.

\subsection{Photo-physics over broadband light excitation}

In order to gain a more complete understanding of the photo-physics in solar cells, we also examined the photoluminescence kinetics over a broad spectral range of excitation energies, 1.8 and $2.7 \mathrm{eV}$ (Figure 5a-c), while keeping the excess carrier density close to 1 solar irradiance. First, the material was investigated in the bimolecular regime (Figure 5a, see also Figure 1b) and 


\section{WILEY-VCH}

a pure bimolecular recombination process was observed with negligible trap-assisted decay to deep trap states (Figure 5c, black square symbols). The quality of the fits were characterized by $\mathrm{R}^{2} \geq 0.8$ and were mainly impaired by the inevitable noise in the data at such low photon flux densities (Figure S5). The bimolecular coefficients were found to range from $\approx 5.3 \times 10^{-9}$ to less than $0.5 \times 10^{-9} \mathrm{~cm}^{3} / \mathrm{s}$ (Figure $5 \mathrm{~b}$, see also Figure S5). The error in the determination of these values is mainly related to the maximum 5\% error on the absorption measurements. As shown in Figure $5 \mathrm{~b}$ the bimolecular recombination coefficient depends strongly on the magnitude of the absorption coefficient and shows an anti-correlated behavior with the absorption coefficient.

Next, we investigated the time-resolved photoluminescence response in the mixed regime and fit the data with equations (1). In contrast to the bimolecular coefficient showing a strong spectral dependence, the trapping rate shows little spectral dependence (Figure 5c, blue round symbols), with a mean value of $\sim 4 \times 10^{7} \mathrm{~s}^{-1}$. This suggests that the trap states probed over the whole spectral range of photo-excitation are of similar nature.

Finally, the photoluminescence of several large-area grains under monochromatic excitation was measured over a broad spectral range. Within a single large grain, a standard photoluminescence excitation (PLE) map and the corresponding integrated PL normalized to the absorption coefficient (equivalent to probing the relative change of the quantum yield) are displayed in Figure 5d. Fluctuations in intensity are in the maximum 5\% error related to measurements of the absorption coefficient. The emission spectrum for each excitation energy was acquired by keeping the photo-excited density of carriers at approximately 1 sun of irradiance in order to reflect the conditions of illumination in photovoltaics. Over the entire range, no significant shift or broadening of the emission peak was observed, whereas a strong reduction of the PL intensity occurs towards the blue spectral region following a similar anti- 


\section{WILEY-VCH}

correlated behaviour in comparison to the absorption profile as observed for the bimolecular coefficient.

The spectral profiles of the quantum yield and kinetic parameters are possibly explained by an increased amount of trapping events, that lead to an overall reduction in $\gamma_{b}$ due to a dominating but slower non-radiaZtive bimolecular decay, taking place at high energy during intra-band relaxation of carriers (Figure 6, middle-right panel). This hypothesis was drawn from both the similar nature of trap states across the visible spectrum and the broadband absorption mechanisms as described by Even et al. ${ }^{[35,40,41]}$ (also sketched in Figure 6, left panel). Qualitatively, we describe the intra-band relaxation in reciprocal space via the differential equation: $n(k)=-P_{i} n(k)$, with $n(k)$ the carrier density at wave vector $k$ and $P_{i}$ the probability of trapping carriers in k-space (assumed to be constant). Using the band dispersion relation $E=$ $\frac{\hbar k^{2}}{2 m^{*}}$ along the R-M direction in k-space, where the $m^{*}$ is the effective mass ${ }^{[42,43]}$, the carrier density at the R-point following excitation at $\mathrm{E}(\mathrm{k})$ is proportional to exp $\left[-P_{i} \sqrt{2 m^{*} E / \hbar}\right]$, which yields a reasonable qualitative fit to the integrated PL (Figure 5d, dashed line). From above spectral dependent TRPL results, we estimated an upper limit of the trap saturation threshold density $N_{\text {th }}$ over a broad spectral range (Figure S6), demonstrating that passivation of shallow trap states was observed at values below solar excitation density for photons energies below $\sim 2$ $\mathrm{eV}$, however at slightly higher values above $2 \mathrm{eV}$. This observation corroborates the higher probability for carriers generated with blue-light excitation to undergo trap-assisted recombination as they are exposed to a higher density of trap states, both shallow and deep, distributed along the bands in k-space while undergoing intra-band relaxation (schematic in Figure 6). In addition to this effect, we can expect surface trap states, self-trapped charges or self-trapped excitons, originating from electron-phonon coupling at the thin film surface ${ }^{[44]}$, to be 


\section{WILEY-VCH}

prevalent under blue-light excitation which probes a more shallow layer of the thin film (see also Figure S7).

\subsection{Solar cell performance}

To confirm that trap-assisted recombination is not an influential process in a high qualityoperating device, we investigated the performance of a planar solar cell (see inset in Figure 7b) with the same material active region as above over a wide range of illumination irradiance. We also probed the recombination of photo-generated carriers by measuring $V_{\mathrm{OC}}$ as a function of light intensity, Figure 7a. In this measurement, all of the carriers recombine in the active layer because there is no net current flow. Here the slope of $V_{O C} v s$ irradiance on a linear-log scale determines the so-called ideality factor $n$. More precisely, $n k_{B} T / q$ is the slope of the linear curve where $q$ the elementary charge. When $n=1$ the measurement suggests that bimolecular recombination is the dominating decay mechanism in the active layer ${ }^{[45]}$. Larger values up to $n=2$ indicate trap-assisted recombination is prevalent and the material has a relatively large density of deep level electronic impurities that mask bimolecular recombination. For this device and material we found $n \sim 1.1$ where the small deviation from an ideality of 1 is expected to result from the presence of a few grain boundaries under the contact and illumination area ${ }^{[2]}$. This confirms that bimolecular recombination is the dominating dynamics for carriers in this material at macroscopic scale, as well as that decay of carriers to deep electronic defects is minor and does not alter the overall transport properties of devices. Moreover, both the micro- and macro-scale carrier dynamics results are reflected in the external quantum efficiency under one sun illumination that yields values larger than $80 \%$ above the band-gap, Figure $7 \mathrm{~b}$. This clearly suggests few losses after absorption and nearly $100 \%$ internal quantum efficiency for single crystals, in agreement with recent studies ${ }^{[21,46,47]}$. 


\section{WILEY-VCH}

\subsection{Microscopic origins of photovoltaic efficiency}

Knowledge of the underlying physics of shallow trap passivation, bimolecular recombination, and spectral variations of the photoluminescence kinetics and intensity are important for understanding the transport properties of large grain perovskite semiconductors. Using Langevin theory describing the recombination of free carriers in diffusion limited semiconductors, the spectral and excitation density dependence of the effective diffusion constant of carriers can be derived as $D=\frac{\gamma_{b}}{4 \pi r_{\mathrm{C}}}$ (Figure S5), using the Onsager radius $r_{\mathrm{c}}=\frac{k_{c} q^{2}}{\varepsilon_{S} k_{B} T}$, where $k_{C}$ is the Coulomb constant, $\varepsilon_{0}$ the vacuum permittivity, and $\varepsilon_{S}=35$ the static dielectric constant ${ }^{[48]}$ (see details in Note S4). Values of $D$ are in the range $\sim 0.1-2.5 \times 10^{-3} \mathrm{~cm}^{2} / \mathrm{s}$ depending on excitation energy, with similar spectral dependence as $\gamma_{b}$. From the values of diffusion constant, at room temperature, we expect minority carrier mobility in the range $\sim 10^{-1}-10^{-2} \mathrm{~cm}^{2} / \mathrm{V}$.s, with a similar spectral profile as the diffusion constant and $\gamma_{b}$. These values are of same magnitude as those reported for bulk $\mathrm{NH}_{3} \mathrm{CH}_{3} \mathrm{PbI}_{3}$ (see refs. ${ }^{[49,50]}$ and literature therein), however much smaller than what is expected theoretically ${ }^{[49,51]}$. Using derived mobilities from the bi-molecular coefficients, the Nernst-Townsend-Einstein relation would yield un-physical sub-fs elastic scattering mean free times, assuming effective masses reported in the literature ${ }^{[42,43]}$. Thus, we infer in these systems there may be an effective mass enhancement due to electron-phonon coupling linked to polaronic states $^{[52-54]}$, or carrier localization effects ${ }^{[36]}$, mostly caused by an orientational disorder of organic cation. In fact, it is understood that the orientational disorder of the organic cation (methylammonium ions) in the lattice is altered by polarized light, which can give rise to local structural distortions that cause changes in the electronic and vibrational landscapes of perovskites ${ }^{[52,55-59]}$. For example, this may be reflected in the recent observation of giant photoinduced dielectric constants ${ }^{[60]}$ and Glass coefficients ${ }^{[55]}$, that increase linearly with broadband 


\section{WILEY-VCH}

excitation intensity. Since these effects increase with increasing excitation energy the overall screening of charge carriers may become more effective reducing the probability of recombination, which is reflected by reductions in $\gamma_{b}$ and $D$.

Further, lower-limit values of mobility predicted from first principle $\left(140 \mathrm{~cm}^{2} / \mathrm{V} . \mathrm{s}\right.$ for holes and $\left.466 \mathrm{~cm}^{2} / \mathrm{V} . \mathrm{s}\right)^{[49]}$ yield a Langevin bimolecular coefficient ${ }^{[61]}$ of $\gamma_{L}=3.1 \times 10^{-5} \mathrm{~cm}^{3} / \mathrm{s}$. This provides a lower limit for the perovskites transport figure of merit which reflects a suppression of electron-hole recombination, $\frac{\gamma_{b}}{\gamma_{L}} \sim 1.7 \times 10^{-4}$. Using typical mobilities in our solar cells $^{[2,62]}, \sim 5 \mathrm{~cm}^{2} /$ V.s or more for $15 \%$ power conversion efficiency, we find $\gamma_{L}=5.2 \times 10^{-7}$ $\mathrm{cm}^{3} / \mathrm{s}$ and $\frac{\gamma_{b}}{\gamma_{L}}<1 \times 10^{-2}$. This implies that perovskite solar cells fabricated in this fashion operate in the non-Langevin recombination regime, which is necessary for efficient photovoltaic devices with low charge carrier mobilities in order to prevent significant losses due to recombination competing with the charge extraction processes ${ }^{[61]}$. In other words, the lifetime of the charge carriers must be longer than their transit time in the intrinsic electric field in order to obtain efficient energy conversion in solar cells. This can be reformulated in terms of a mobility lower limit as $\mu>\frac{n_{0} \gamma_{b} d^{2}}{V_{\mathrm{bi}}}$, where $V_{\mathrm{bi}}$ is the built-in electric field potential $\left(V_{\mathrm{bi}}=0.78 \mathrm{~V}\right.$ in our solar cells $^{[62]}$ ) with thickness $d=450 \mathrm{~nm}$. We estimate $\mu>0.01 \mathrm{~cm}^{2} / \mathrm{Vs}$ is necessary for efficient energy conversion. These conditions are in good agreement with our TRPL analysis, and fulfilled in our large-area grain perovskite material. This rational further explains the high performances observed in perovskite-based solar cells beyond having low deep level defect densities.

\section{Conclusions}




\section{WILEY-VCH}

Our findings of pure bimolecular recombination are in stark contrast with previous reports on bulk organometallic perovskite materials showing nearly exponential mono-molecular processes at equivalent carrier densities or a combination of both bimolecular recombination and trapassisted relaxation ${ }^{[10,12,14,15,39,63]}$. The origin of this discrepancy may result from small grains where crystal boundaries and defects result in dynamics that deviate from bimolecular recombination because of monomolecular trap assisted recombination ${ }^{[2]}$, Figure $4 \mathrm{~b}$ and Figure S8. Notably, the observation of a pure bimolecular decay is unique for semiconducting solutionprocessed materials at this excitation density as trap-assisted or exciton recombination (both mono-molecular kinetics) are usually dominating processes. Most importantly, our findings bridge the gap between the micro-scale optical properties and macro-scale solar cell performance of crystalline methylammonium halide thin films. These results clearly demonstrate that devices based on solution processed thin film semiconductors can be operated in regimes free of impurities that degrade transport and alter optical properties. More broadly this work asserts the rapid progress in organic-inorganic perovskite based optoelectronics where experimentally determined intrinsic properties represent a clear departure from macroscopic ensembles of nanoparticle systems that are plagued by inhomogeneity and defects. Nevertheless, the mobility of charge carriers in perovskites remains significantly lower than conventional semiconductors, reflecting transport may be similar to that in organic systems without large deep level defect densities associated with multiple interfaces. Further understanding of the details of charge carrier localization and mobilities associated with the dynamic perovskite lattice ${ }^{[64]}$ will aid in providing a physical picture of transport in these hybrid systems and pave the way for improved stability and performance in optoelectronic devices. 


\section{Supporting Information}

Supporting Information is available from the Wiley Online Library or from the author.

\section{Acknowledgements}

This work was supported the Los Alamos National Laboratory LDRD program. L.C.

acknowledges additional support from INCa-Canceropole GSO.

Competing financial interest The authors declare no competing financial interests.

Received: ((will be filled in by the editorial staff))

Revised: ((will be filled in by the editorial staff))

Published online: ((will be filled in by the editorial staff))

[1] S. D. Stranks, H. J. Snaith, Nat. Nanotechnol. 2015, 10, 391.

[2] W. Nie, H. Tsai, R. Asadpour, J.-C. Blancon, A. J. Neukirch, G. Gupta, J. J. Crochet, M.

Chhowalla, S. Tretiak, M. A. Alam, H.-L. Wang, A. D. Mohite, Science 2015, 347, 522.

[3] Z.-K. Tan, R. S. Moghaddam, M. L. Lai, P. Docampo, R. Higler, F. Deschler, M. Price,

A. Sadhanala, L. M. Pazos, D. Credgington, F. Hanusch, T. Bein, H. J. Snaith, R. H. Friend, Nat. Nanotechnol. 2014, 9, 687.

[4] G. Xing, N. Mathews, S. S. Lim, N. Yantara, X. Liu, D. Sabba, M. Grätzel, S.

Mhaisalkar, T. C. Sum, Nat. Mater. 2014, 13, 476.

[5] H. Zhu, Y. Fu, F. Meng, X. Wu, Z. Gong, Q. Ding, M. V. Gustafsson, M. T. Trinh, S. Jin, X.-Y. Zhu, Nat. Mater. 2015, 14, 636.

[6] F. Deschler, M. Price, S. Pathak, L. E. Klintberg, D.-D. Jarausch, R. Higler, S. Hüttner, T. Leijtens, S. D. Stranks, H. J. Snaith, M. Atatüre, R. T. Phillips, R. H. Friend, J. Phys. Chem. Lett. 2014, 5, 1421.

[7] M. D. Bastiani, V. D’Innocenzo, S. D. Stranks, H. J. Snaith, A. Petrozza, APL Mater. 2014, 2, 081509. 


\section{WILEY-VCH}

[8] V. D’Innocenzo, A. R. Srimath Kandada, M. De Bastiani, M. Gandini, A. Petrozza, $J$. Am. Chem. Soc. 2014, 136, 17730.

[9] D. W. deQuilettes, S. M. Vorpahl, S. D. Stranks, H. Nagaoka, G. E. Eperon, M. E. Ziffer, H. J. Snaith, D. S. Ginger, Science 2015, 348, 683.

[10] S. D. Stranks, G. E. Eperon, G. Grancini, C. Menelaou, M. J. P. Alcocer, T. Leijtens, L. M. Herz, A. Petrozza, H. J. Snaith, Science 2013, 342, 341.

[11] G. Xing, N. Mathews, S. Sun, S. S. Lim, Y. M. Lam, M. Grätzel, S. Mhaisalkar, T. C. Sum, Science 2013, 342, 344.

[12] C. Wehrenfennig, G. E. Eperon, M. B. Johnston, H. J. Snaith, L. M. Herz, Adv. Mater. Deerfield Beach Fla 2014, 26, 1584.

[13] C. Wehrenfennig, M. Liu, H. J. Snaith, M. B. Johnston, L. M. Herz, J. Phys. Chem. Lett. 2014, 5, 1300.

[14] Y. Yamada, T. Nakamura, M. Endo, A. Wakamiya, Y. Kanemitsu, J. Am. Chem. Soc. 2014, 136, 11610.

[15] S. D. Stranks, V. M. Burlakov, T. Leijtens, J. M. Ball, A. Goriely, H. J. Snaith, Phys. Rev. Appl. 2014, 2, 034007.

[16] T. C. Sum, N. Mathews, Energy Environ. Sci. 2014, 7, 2518.

[17] J. A. Christians, J. S. Manser, P. V. Kamat, J. Phys. Chem. Lett. 2015, 6, 2086.

[18] M. Saba, M. Cadelano, D. Marongiu, F. Chen, V. Sarritzu, N. Sestu, C. Figus, M. Aresti, R. Piras, A. Geddo Lehmann, C. Cannas, A. Musinu, F. Quochi, A. Mura, G. Bongiovanni, Nat. Commun. 2014, 5, 5049.

[19] A. Marchioro, J. Teuscher, D. Friedrich, M. Kunst, R. van de Krol, T. Moehl, M. Grätzel, J.-E. Moser, Nat. Photonics 2014, 8, 250. 


\section{WILEY-VCH}

[20] J. S. Manser, P. V. Kamat, Nat. Photonics 2014, 8, 737.

[21] Q. Lin, A. Armin, R. C. R. Nagiri, P. L. Burn, P. Meredith, Nat. Photonics 2015, 9, 106.

[22] M. Anaya, G. Lozano, M. E. Calvo, W. Zhang, M. B. Johnston, H. J. Snaith, H. Míguez, J. Phys. Chem. Lett. 2014, 6, 48.

[23] P. Löper, M. Stuckelberger, B. Niesen, J. Werner, M. Filipič, S.-J. Moon, J.-H. Yum, M. Topič, S. De Wolf, C. Ballif, J. Phys. Chem. Lett. 2015, 6, 66.

[24] G. Lasher, F. Stern, Phys. Rev. 1964, 133, A553.

[25] E. Yablonovitch, Phys. Rev. Lett. 1987, 58, 2059.

[26] J. Liu, O. V. Prezhdo, J. Phys. Chem. Lett. 2015, 4463.

[27] Y. Jiang, M. A. Green, R. Sheng, A. Ho-Baillie, Sol. Energy Mater. Sol. Cells 2015, 137, 253.

[28] A. Dmitriev, A. Oruzheinikov, J. Appl. Phys. 1999, 86, 3241.

[29] Y. P. Varshni, Phys. Status Solidi B 1967, 19, 459.

[30] P. Asbeck, J. Appl. Phys. 1977, 48, 820.

[31] Y. Yamada, T. Yamada, L. Q. Phuong, N. Maruyama, H. Nishimura, A. Wakamiya, Y. Murata, Y. Kanemitsu, J. Am. Chem. Soc. 2015, 137, 10456.

[32] E. D. Kosten, B. M. Kayes, H. A. Atwater, Energy Environ. Sci. 2014, 7, 1907.

[33] W. E. I. Sha, X. Ren, L. Chen, W. C. H. Choy, Appl. Phys. Lett. 2015, 106, 221104.

[34] A. Miyata, A. Mitioglu, P. Plochocka, O. Portugall, J. T.-W. Wang, S. D. Stranks, H. J. Snaith, R. J. Nicholas, Nat. Phys. 2015, 11, 582.

[35] J. Even, L. Pedesseau, C. Katan, J. Phys. Chem. C 2014, 118, 11566.

[36] J. Ma, L.-W. Wang, Nano Lett. 2015, 15, 248.

[37] J. Kim, S.-H. Lee, J. H. Lee, K.-H. Hong, J. Phys. Chem. Lett. 2014, 5, 1312. 


\section{WILEY-VCH}

[38] W.-J. Yin, T. Shi, Y. Yan, Appl. Phys. Lett. 2014, 104, 063903.

[39] E. M. Hutter, G. E. Eperon, S. D. Stranks, T. J. Savenije, J. Phys. Chem. Lett. 2015, 6, 3082.

[40] J. Even, J. Phys. Chem. Lett. 2015, 6, 2238.

[41] J. Even, L. Pedesseau, C. Katan, M. Kepenekian, J.-S. Lauret, D. Sapori, E. Deleporte, J. Phys. Chem. C 2015, 119, 10161.

[42] P. Umari, E. Mosconi, F. De Angelis, Sci. Rep. 2014, 4.

[43] M. R. Filip, C. Verdi, F. Giustino, J. Phys. Chem. C 2015.

[44] X. Wu, M. T. Trinh, D. Niesner, H. Zhu, Z. Norman, J. S. Owen, O. Yaffe, B. J. Kudisch, X.-Y. Zhu, J. Am. Chem. Soc. 2015, 137, 2089.

[45] L. J. A. Koster, V. D. Mihailetchi, R. Ramaker, P. W. M. Blom, Appl. Phys. Lett. 2005, $86,123509$.

[46] J. M. Ball, S. D. Stranks, M. T. Hörantner, S. Hüttner, W. Zhang, E. J. W. Crossland, I. Ramirez, M. Riede, M. B. Johnston, R. H. Friend, H. J. Snaith, Energy Environ. Sci. 2015, 8, 602.

[47] B. Yang, O. Dyck, J. Poplawsky, J. Keum, A. Puretzky, S. Das, I. Ivanov, C. Rouleau, G. Duscher, D. Geohegan, K. Xiao, J. Am. Chem. Soc. 2015, 137, 9210.

[48] N. Onoda-Yamamuro, T. Matsuo, H. Suga, J. Phys. Chem. Solids 1992, 53, 935.

[49] X. Y. Chin, D. Cortecchia, J. Yin, A. Bruno, C. Soci, Nat. Commun. 2015, 6, 7383.

[50] F. Li, C. Ma, H. Wang, W. Hu, W. Yu, A. D. Sheikh, T. Wu, Nat. Commun. 2015, 6, 8238.

[51] Y. He, G. Galli, Chem. Mater. 2014, 26, 5394.

[52] J. M. Frost, K. T. Butler, A. Walsh, APL Mater. 2014, 2, 081506. 


\section{WILEY-VCH}

[53] X.-Y. Zhu, V. Podzorov, J. Phys. Chem. Lett. 2015, 4758.

[54] T. M. Brenner, D. A. Egger, A. M. Rappe, L. Kronik, G. Hodes, D. Cahen, J. Phys. Chem. Lett. 2015, 4754.

[55] H. T. Fan Zheng, J. Phys. Chem. Lett. 2014, 6, 31.

[56] C. Motta, F. El-Mellouhi, S. Kais, N. Tabet, F. Alharbi, S. Sanvito, Nat. Commun. 2015, 6,7026 .

[57] A. M. A. Leguy, J. M. Frost, A. P. McMahon, V. G. Sakai, W. Kockelmann, C. Law, X. Li, F. Foglia, A. Walsh, B. C. O’Regan, J. Nelson, J. T. Cabral, P. R. F. Barnes, Nat. Commun. 2015, 6, 7241 .

[58] A. A. Bakulin, A. Rao, V. G. Pavelyev, P. H. M. van Loosdrecht, M. S. Pshenichnikov, D. Niedzialek, J. Cornil, D. Beljonne, R. H. Friend, Science 2012, 335, 1340.

[59] H. Tsai, W. Nie, P. Cheruku, N. H. Mack, P. Xu, G. Gupta, A. D. Mohite, H.-L. Wang, Chem. Mater. 2015, 27, 5570.

[60] R. S. S. Emilio J. Juarez-Perez, J. Phys. Chem. Lett. 2014, 5, 2390.

[61] A. Pivrikas, G. Juška, A. J. Mozer, M. Scharber, K. Arlauskas, N. S. Sariciftci, H. Stubb, R. Österbacka, Phys. Rev. Lett. 2005, 94, 176806.

[62] X. Sun, R. Asadpour, W. Nie, A. D. Mohite, M. A. Alam, IEEE J. Photovolt. 2015, 5, 1389.

[63] G.-J. A. H. Wetzelaer, M. Scheepers, A. M. Sempere, C. Momblona, J. Avila, H. J. Bolink, Adv. Mater. 2015, 27, 1837.

[64] J. M. Frost, K. T. Butler, F. Brivio, C. H. Hendon, M. van Schilfgaarde, A. Walsh, Nano Lett. 2014, 14, 2584.

[65] G. Kresse, J. Furthmüller, Comput. Mater. Sci. 1996, 6, 15. 


\section{WILEY-VCH}

[66] G. Kresse, D. Joubert, Phys Rev B 1999, 59, 1758.

[67] J. P. Perdew, K. Burke, M. Ernzerhof, Phys. Rev. Lett. 1996, 77, 3865.

[68] T. Baikie, Y. Fang, J. M. Kadro, M. Schreyer, F. Wei, S. G. Mhaisalkar, M. Graetzel, T.

J. White, J. Mater. Chem. A 2013, 1, 5628.

[69] M. Cesaria, A. P. Caricato, M. Martino, J. Opt. 2012, 14, 105701.

[70] R. E. Denton, R. D. Campbell, S. G. Tomlin, J. Phys. Appl. Phys. 1972, 5, 852.

[71] J. C. de Mello, H. F. Wittmann, R. H. Friend, Adv. Mater. 1997, 9, 230.

[72] M. Gajdoš, K. Hummer, G. Kresse, J. Furthmüller, F. Bechstedt, Phys. Rev. B 2006, 73, 045112.

[73] K. Tanaka, T. Takahashi, T. Ban, T. Kondo, K. Uchida, N. Miura, Solid State Commun. 2003, 127, 619 .

[74] J. Even, L. Pedesseau, J.-M. Jancu, C. Katan, J. Phys. Chem. Lett. 2013, 4, 2999.

[75] J. Even, L. Pedesseau, J.-M. Jancu, C. Katan, Phys. Status Solidi RRL - Rapid Res. Lett. 2014, 8,31 .

[76] J. R. Bolton, M. D. Archer, J. Phys. Chem. 1991, 95, 8453.

[77] T. Ahmed, C. La-o-vorakiat, T. Salim, Y. M. Lam, E. E. M. Chia, J.-X. Zhu, EPL Europhys. Lett. 2014, 108, 67015. 
(a)

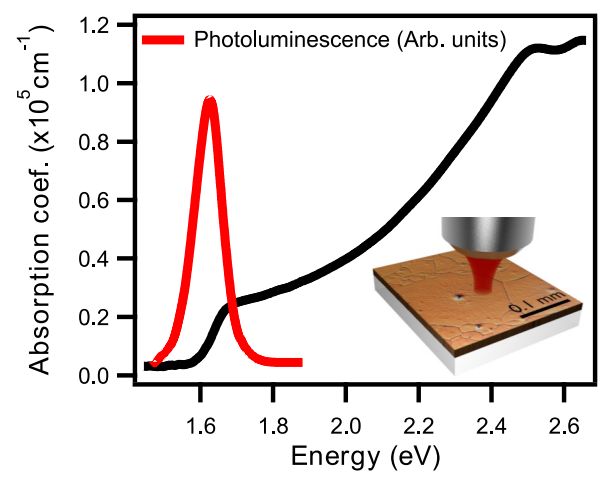

(b)

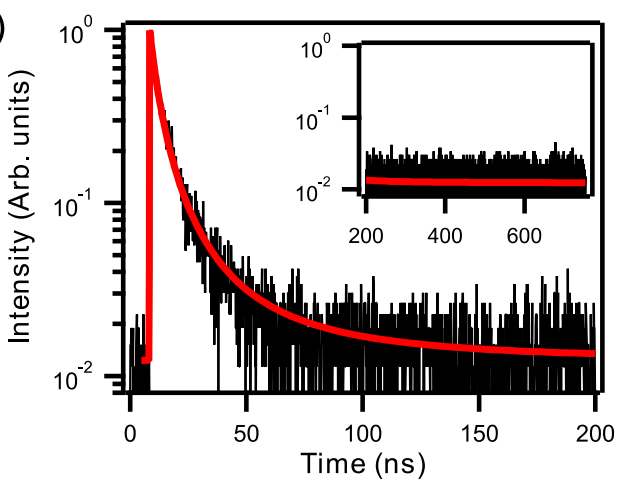

Figure 1. (a) Absolute value of the absorption coefficient (black) and the PL spectrum (red). (inset) Phase contrast microscope image of a large-area grain organometallic perovskite thin film deposited on glass. A schematic of how the confocal microscopy experiment probes a single grain is also shown. (b) Time-correlated single photon counting histogram of the PL (black) and the bimolecular recombination model (red). (inset) Same showing the data at longer times.

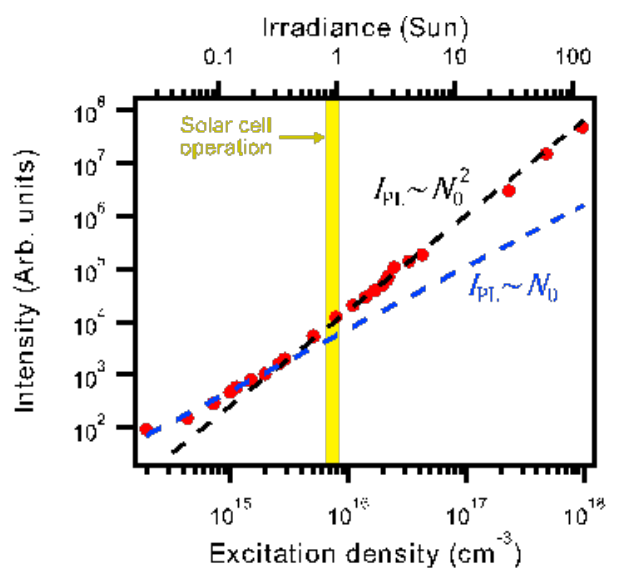


Figure 2. Photoluminescence intensity as a function of the photo-excitation density. Dashed lines are fit of the data.
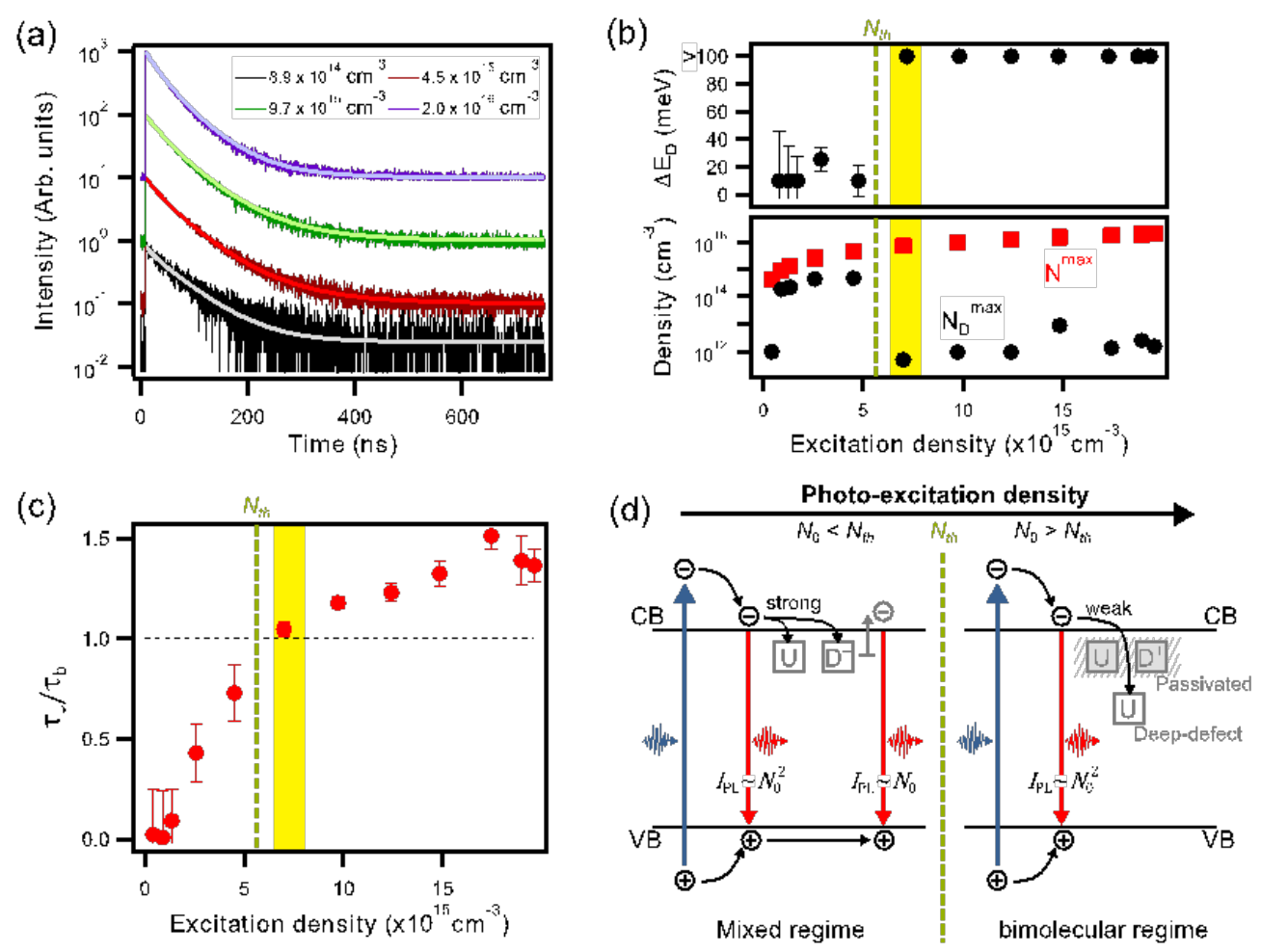

Figure 3. (a) Time-correlated single photon counting histogram of the PL (darken colors) and the recombination model (lighter colors) for different excitation density. Data were acquired using excitation $690 \mathrm{~nm}(1.80 \mathrm{eV})$. (b) $\Delta \mathrm{E}_{\mathrm{D}}$ and maximum density of $\mathrm{N}, \mathrm{N}_{\mathrm{D}}$ as well as (c) lifetimes derived from the fitting of the data as a function of excitation density. The vertical dashed line at the saturation threshold excitation density Nth locates the transition between the mixed regime and the bimolecular regime. (d) Schematic of the main photo-physical processes in perovskites. In the mixed regime, after absorption of a photon, the main relaxation path is via trap-assisted non-radiative recombination to shallow charged (here we illustrate the situation for a donor state $\mathrm{D}^{+}$) or neutral $(\mathrm{U})$ defect. The bimolecular recombination (radiative or nonradiative) involves a photo-generated free hole, which recombines with either a photo-generated 


\section{WILEY-VCH}

free electron (weak) or a bound electron to a donor state (strong). In the bimolecular regime, shallow trap states have been passivated and the main relaxation process is via bimolecular recombination of photo-excited carriers.

(a)

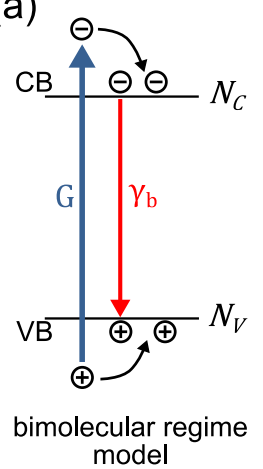

(b)

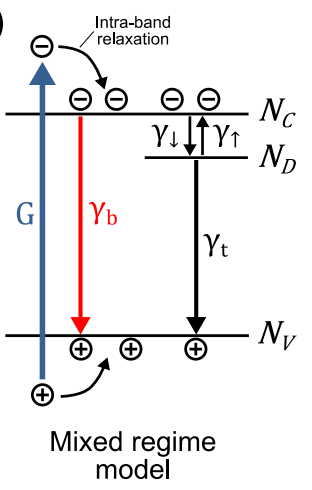

Figure 4. (a) The dynamics of light emission is modelled by an excitation density generation rate provided by the laser $\mathrm{G}$ followed by an unresolved ultrafast relaxation to conduction and valence band edges (top and bottom arrows) preceding a bimolecular recombination of conduction and valence band densities $\left(\mathrm{N}_{\mathrm{C}}, \mathrm{N}_{\mathrm{V}}\right)$ characterized by a single bimolecular coefficient $\gamma_{\mathrm{b}}$. (b) When defects are present, such as an electronic state characterized by a conduction band trap density $\mathrm{N}_{\mathrm{D}}$, a competing relaxation channel $\gamma_{t}$ appears leading to kinetics that alter the bimolecular decay. Carriers can exchange density between the band-edge $\left(\mathrm{N}_{\mathrm{C}}\right)$ and the trap state $\left(\mathrm{N}_{\mathrm{D}}\right)$ as modelled via the rate couple $\left(\gamma_{\downarrow}, \gamma_{\uparrow}\right)$ and the energy separation between $N_{C}$ and $N_{D}$ (noted $\left.\Delta \mathrm{E}_{\mathrm{D}}\right)$. 

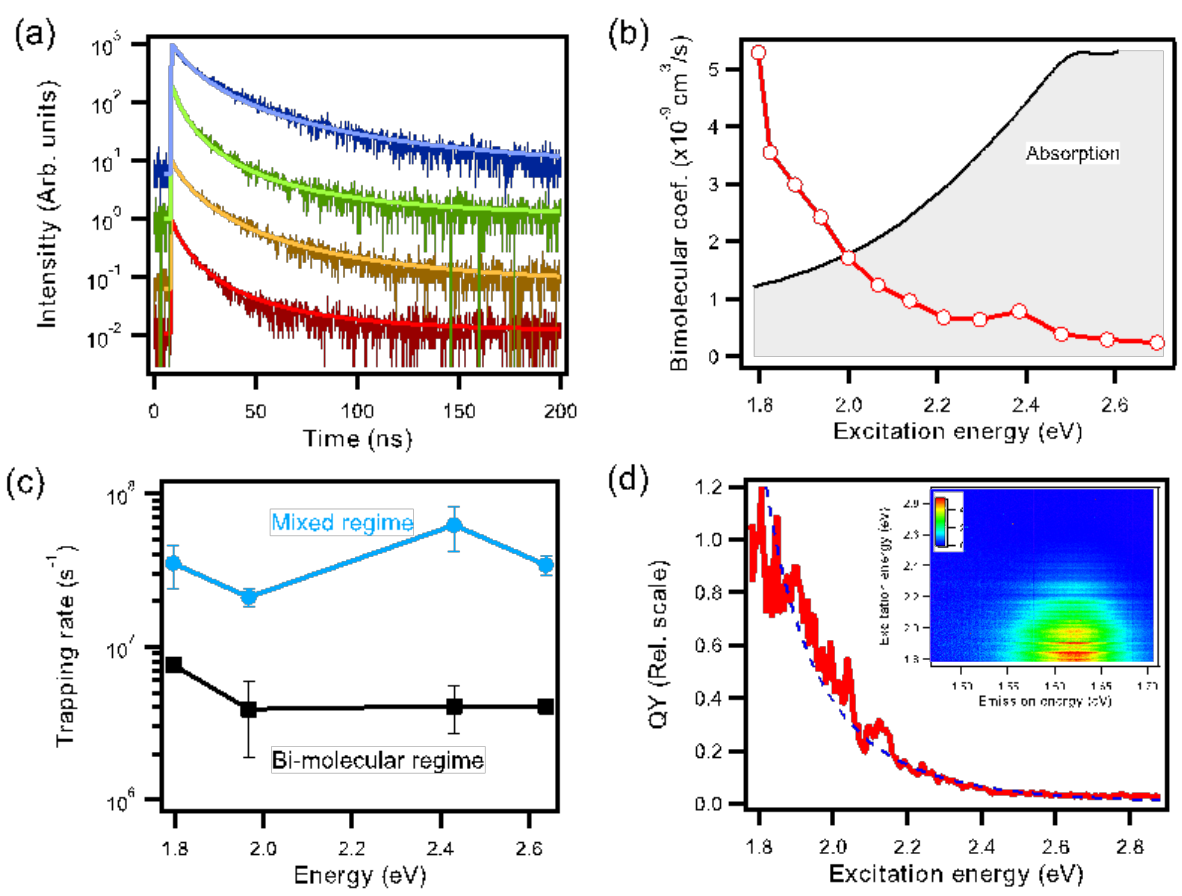

Figure 5. (a) Time-correlated single photon counting histogram of the PL (darken colors) and the bimolecular recombination model (lighter colors) for selected excitation energies, from bottom to top: $1.88 \mathrm{eV}(660 \mathrm{~nm}), 2.14$ (580), 2.38 (520), 2.58 (480). Photo-excitation density was kept in lower part of the bimolecular regime, i.e. trap-assisted non-radiative recombination is negligible. (b) (open symbols) Bimolecular coefficient $\gamma_{\mathrm{b}}$ derived from the fitting of the data, errors from the fits are negligible as compared to the symbol size however the maximum $\sim 5 \%$ coming from the absorption measurements is not displayed. (grey region) Absorption coefficient profile. (c) Trapping rate constants to shallow trap states (mixed regime) and deep trap states (bimolecular regime). (d) Excitation energy dependence of the relative quantum yield (red). Broken curves sketch the fitting of the data with the model described in the main text. (inset) Color-map of the photoluminescence intensity (rel. units) versus excitation (y-axis) and emission (x-axis) energy. 

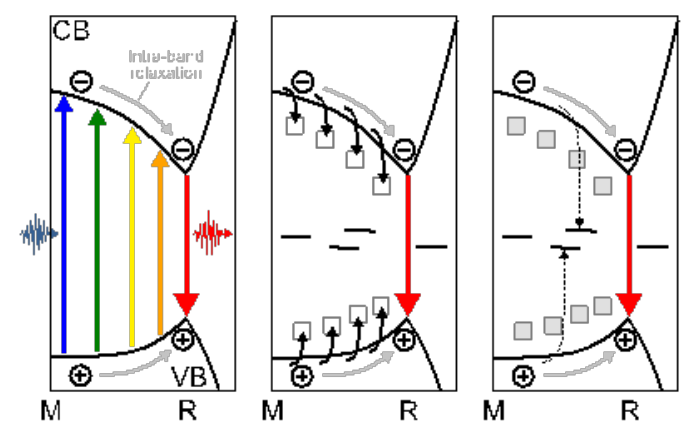

Figure 6. Schematics of the band structure of the high-temperature pseudo-cubic phase of perovskite (from ref. ${ }^{[41]}$ ) during: photoexcitation (left), relaxation in the mixed regime (middle), and relaxation in the bimolecular regime (right). Optical excitations generate free carriers in the valence $\left(\mathrm{E}_{1 / 2, \mathrm{~g}}\right)$ and conductions $\left(\mathrm{E}_{1 / 2, \mathrm{u}}\right)$ bands which relax towards the $\mathrm{R}$ point where the emission takes place.

(a)

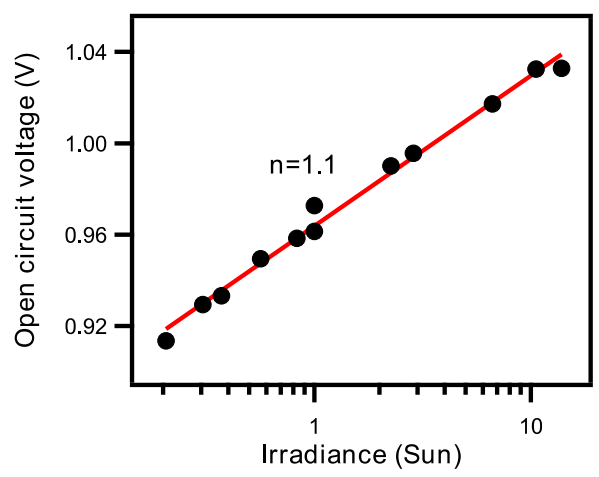

(b)

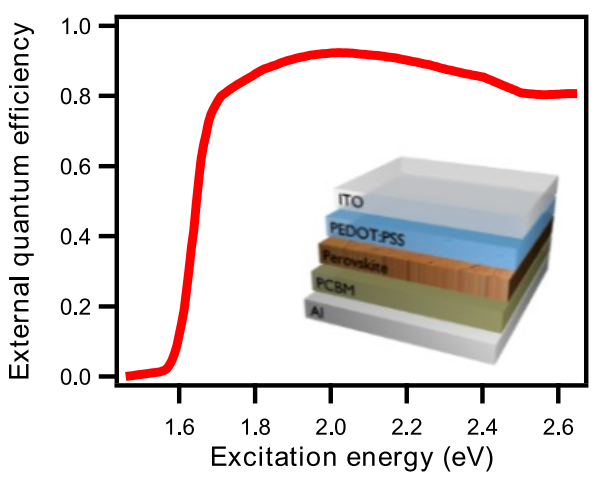

Figure 7. (a) Open circuit voltage as a function of irradiance (filled black circles). The line is a fit showing an ideality factor close to 1 suggesting bimolecular recombination of photo- 


\section{WILEY-VCH}

generated carriers. (b) Spectrally resolved absolute value of the external quantum efficiency measured on a full device. The inset is a schematic of the device geometry. 


\section{WILEY-VCH}

1. Introduction

2. Results and Discussion

2.1. Unambiguous bimolecular recombination in large grain perovskites

2.2. Density dependent emission intensity and dynamics

2.3. Photo-physics over broadband light excitation

2.4. Solar cell performance

2.5. Microscopic origins of photovoltaic efficiency

\section{Conclusion}

Keywords: Electronic Processes, Organic-Inorganic Perovskite Semiconductors, Photoluminescence, Photovoltaic Devices.

J.-C. Blancon, W. Nie, A. J. Neukirch, G. Gupta, S. Tretiak, L. Cognet, A. D. Mohite, J. J. Crochet*

The effects of electronic impurities and electron-hole recombination dynamics on largegrain organic-inorganic perovskite photovoltaic efficiencies 